\title{
Research article \\ Improving the existing cardiovascular risk scoring systems for better prediction in type 2 diabetes mellitus with and without coronary artery disease: A cross sectional study
}

\author{
Arpita Chakraborty ${ }^{1}$, M. Mukhyaprana Prabhu' ${ }^{2}$, Weena Stanley ${ }^{3}$ \\ ${ }^{1}$ Research Scholar, ${ }^{2}$ Professor, ${ }^{3}$ Associate Professor, Department of General Medicine, Kasturba Medical College Manipal, \\ Manipal Academy of Higher Education, Manipal 576 104, Karnataka, India
}

\author{
(Received: January $2021 \quad$ Revised: June $2021 \quad$ Accepted: June 2021)
}

Corresponding author: Weena Stanley.Email: weena.stanley@manipal.edu

\begin{abstract}
Introduction and Aim: Atherosclerotic cardiovascular disease (ASCVD) and Framingham score (FRS) are two important scores used for the prediction of coronary events in an individual. Both of these scores do not account for certain known predisposing factors such as BMI, duration of diabetes, LDL cholesterol and glycemic index levels among type 2 diabetes mellitus (T2DM) subjects. So, this study aims to assess whether addition of any of these important risk factors might help in improving these scoring systems in T2DM patients.

Methodology: Our study is a cross-sectional study which included 320 T2DM patients without CAD and 120 T2DM patients with CAD. Duration of T2DM, BMI, glycated hemoglobin and fasting lipid profile values of the T2DM patients were recorded from the Laboratory information system.

Results: IBM Statistical Package for the Social Sciences (SPSS) 16 version was used for statistical analysis. T2DM patients with CAD had more years of duration of T2DM than the patients without CAD and a significant association was found $\left(\mathrm{p}=.045^{*}\right)$. A strong significant positive correlation was observed between FRS score and duration of T2DM in diabetic patients with CAD ( $\mathrm{r}=.331, \mathrm{p}=<.0001 *)$.

Conclusion: Our observations imply that inclusion of important parameter such as duration of T2DM might improve in better calculation of these risk scores in T2DM patients. Future studies are needed to assess the performance of existing risk scores by including important parameter such as duration of diabetes which might help in improving these scoring systems in T2DM patients.
\end{abstract}

Keywords: Atherosclerotic cardiovascular disease; duration of T2DM; Framingham score; lipids; type 2 diabetes mellitus.

\section{INTRODUCTION}

A mong the diabetic population deaths and hospitalizations take place predominantly due to coronary artery disease (CAD) $(1,2)$. The major determinant which plays role in the prognosis of patients with CAD is prolonged duration of T2DM. In patients suffering from T2DM there is a probability of 2-4 folds increase in the mortality rate from CAD and worse prognosis (3). The coronary risk in an individual can be estimated with various risk scores such as "Framingham risk core of coronary heart disease and cardiovascular disease, QRISK2, Joint British Society risk calculator 3 (JBS3), Atherosclerotic Cardiovascular Disease (ASCVD), United Kingdom Prospective Diabetes Study (UKPDS) and WHO risk charts" (2). An Indian study showed the FRS-CVD risk assessment tool to be the best among all the available cardiovascular risk assessment tools with prediction rate of $51.9 \%$ whereas WHO risk charts and ASCVD risk tool were found to be the worst with prediction rates ranging from $16.2 \%$ to $28.3 \%$ (4).

Both the ASCVD and FRS scores include gender, ethnicity, total cholesterol (TC) levels, High Density Lipoprotein (HDL) cholesterol levels, systolic blood pressure (SBP), smoking habit, presence or absence of
T2DM and treatment for hypertension. But it does not account for other known predisposing factors such as the duration of diabetes, glycemic control, and LowDensity Lipoprotein (LDL) cholesterol. The main difference which lies in the calculator between the FRS and ASCVD score calculators is the presence of an extra risk factor in FRS which is whether the person is suffering from CVD, peripheral vascular disease and stroke. The Framingham heart study stated that duration of diabetes plays a significant role and elevates the risk of deaths due to cardiovascular disease irrespective of other co morbidities (5). In the diabetic population, glycated haemoglobin $(\mathrm{HbA} 1 \mathrm{c})$ contributes a vital role and is probably the most important risk factor which leads to cardiovascular mortality. Cluster of changes in the plasma lipid or lipoproteins levels in the blood such as diminished levels of HDL cholesterol, preponderance levels of small dense LDL cholesterol, and elevated levels of triglyceride had strong association with T2DM and insulin resistance (6). Thus, this study aims to assess whether addition of any of these important risk factors might help in improving these scoring systems in T2DM patients. 


\section{METHODOLOGY}

\section{Study design}

This is a cross sectional study which was carried out at a tertiary care hospital in coastal Karnataka, South India.

\section{Sample size}

After obtaining clearance from ethics committee (IEC 715/2018), consented 320 T2DM patients between the age 40-74 years without CAD and 120 T2DM patients of the same age group with CAD were recruited for the study.

\section{Procedure}

Demographic details, treatment history, lab investigations like glycated haemoglobin (HbA1c), fasting blood sugar (FBS), post prandial blood sugar (PPBS) and fasting lipid profile (FLP) were recorded. HbA1c levels were measured using turbidimetric inhibition immunoassay method, FBS and PPBS (Hexokinase method), Total Cholesterol (Cholesterol oxidase peroxidase (CHOD-POD) method), Triacylglycerol (GPO Trinder method), HDL cholesterol (directed homogeneous method) and LDL cholesterol (enzymatic method). Total cholesterol, HDL levels and smoking status were not considered for the statistical analysis as all are included in calculating both FRS and ASCVD scores. ASCVD score was calculated by ASCVD score estimator developed by American College of Cardiology "(https://qxmd.com/calculate/calculator 37/acc-ahacv-risk-calculator-2013)" and the Framingham risk score was calculated by using Framingham risk calculator developed by Framingham Heart Study “(https://qxmd.com/calculate/calculator_252/framing ham-risk-score-2008)" in both T2DM patients with and without CAD.

\section{Data analysis}

IBM Statistical Package for the Social Sciences (SPSS) 16 version was used for statistical analysis. Demographic parameters and the clinical variables that were not captured in both the ASCVD and FRS scores were compared within the two groups i.e., T2DM patients with and without CAD. Mann Whitney test was used for comparing the demographic parameters between both these groups. Spearman correlation was used to correlate laboratory parameters with respective scores in each group. Statistical significance was considered when $p$ values were $<0.05$ ( 2 -tailed).

\section{RESULTS}

Total 440 subjects were divided into two categories namely T2DM patients without CAD $(n=320)$ and with CAD $(n=120)$. T2DM patients with CAD had more years of duration of T2DM than the ones without $\mathrm{CAD}$ and a significant association was found $(\mathrm{p}=.045$; Table 1). Also, a significant strong positive correlation was found between FRS score and duration of T2DM in T2DM patients with CAD $(\mathrm{r}=.331, \mathrm{p}=<.0001)$. Weak positive correlation was found between FRS score and LDL cholesterol ( $\mathrm{r}=.114, \mathrm{p}=.042)$ and between ASCVD score and duration of T2DM $(\mathrm{r}=.278, \mathrm{p}=<.0001)$ in T2DM patients with CAD. Weak negative correlation was found between ASCVD score and BMI ( $\mathrm{r}=-.130, \mathrm{p}=.020)$ in T2DM patients with CAD (Table 2). However, no correlation was found between any of the parameters with both of these scoring systems in T2DM patients without CAD (Table 3).

Table1: Comparison of clinical variables and demographic parameters in T2DM patients with and without CAD

\begin{tabular}{|c|c|c|c|}
\hline Name of variables & $\begin{array}{c}\text { T2DM patients without CAD } \\
(\mathbf{n = 3 2 0})\end{array}$ & $\begin{array}{c}\text { T2DM patients with CAD } \\
(\mathbf{n = 1 2 0})\end{array}$ & *p value \\
\hline BMI $\left(\mathrm{Kg} / \mathrm{m}^{2}\right)$ & $25.35(23.02,28.9)$ & $25.95(22.7,28.5)$ & .752 \\
\hline LDL cholesterol $(\mathrm{mg} / \mathrm{dl})$ & $85.5(64,123)$ & $89(73,109.5)$ & .396 \\
\hline Duration of T2DM (years) & $7(3,10)$ & $8(5,10)$ & $\mathbf{0 4 5}$ \\
\hline $\mathrm{HbA}_{1} \mathrm{c}(\mathrm{mg} / \mathrm{dl})$ & $7.5(6.62,9.1)$ & $8(7,8)$ & .139 \\
\hline
\end{tabular}

*Mann Whitney test

Table 2: Correlation of demographic parameters with FRS and ASCVD scores among T2DM patients with CAD $(\mathrm{n}=120)$

\begin{tabular}{|c|c|c|}
\hline Name of variables & r value & *p value \\
\hline FRS score and duration of T2DM (years) & .331 & $<.0001$ \\
\hline FRS score and BMI $\left(\mathrm{Kg} / \mathrm{m}^{2}\right)$ & -.098 & .081 \\
\hline FRS score and LDL $(\mathrm{mg} / \mathrm{dl})$ & .114 & $\mathbf{. 0 4 2}$ \\
\hline FRS score and $\mathrm{HbA}_{1} \mathrm{c}(\mathrm{mg} / \mathrm{dl})$ & .028 & .618 \\
\hline ASCVD score and duration of T2DM (years) & .278 & $<.0001$ \\
\hline ASCVD score and BMI $\left(\mathrm{Kg} / \mathrm{m}^{2}\right)$ & -.130 & $\mathbf{. 0 2 0}$ \\
\hline ASCVD score and LDL $(\mathrm{mg} / \mathrm{dl})$ & .020 & .720 \\
\hline ASCVD score and $\mathrm{HbA}_{1} \mathrm{c}(\mathrm{mg} / \mathrm{dl})$ & -.020 & .728 \\
\hline
\end{tabular}

* Spearman correlation 
Table 3: Correlation of demographic parameters with FRS score and ASCVD scores among T2DM patients without CAD $(n=320)$

\begin{tabular}{|c|c|c|}
\hline Name of variables & r value & *p value \\
\hline FRS score and duration of T2DM (years) & .131 & .155 \\
\hline FRS score and BMI $\left(\mathrm{Kg} / \mathrm{m}^{2}\right)$ & -.075 & .414 \\
\hline FRS score and LDL $(\mathrm{mg} / \mathrm{dl})$ & -.031 & .740 \\
\hline FRS score and $\mathrm{HbA}_{1} \mathrm{c}(\mathrm{mg} / \mathrm{dl})$ & .031 & .734 \\
\hline ASCVD score and duration of T2DM (years) & .178 & .052 \\
\hline ASCVD score and BMI $\left(\mathrm{Kg} / \mathrm{m}^{2}\right)$ & -.044 & .632 \\
\hline ASCVD score and LDL $(\mathrm{mg} / \mathrm{dl})$ & -.070 & .450 \\
\hline ASCVD score and $\mathrm{HbA}_{1} \mathrm{c}(\mathrm{mg} / \mathrm{dl})$ & -.059 & .520 \\
\hline
\end{tabular}

*Spearman correlation

\section{DISCUSSION}

Our study showed significant association and strong positive correlation between duration of T2DM and FRS score in T2DM patients with CAD. In the Framingham heart study (FHS) duration of T2DM was found to be a major risk factor for causing deaths due to coronary heart disease (5) which is similar to the finding of our study. A duration of four-year risk was found among the pre-diabetic ranging from 12.7-22.3 times risk elevation in males and females respectively in the FHS study (7). In one study the risk of developing coronary heart disease was found to be 1.38 -fold more for every ten years increment in duration of T2DM (95\% CI 0.99-1.92) and the risk associated for developing coronary heart disease mortality was 1.86 -fold more (1.17-2.93) for the same increment in duration of T2DM which is also similar to the finding of our study (5). This might be due to the fact that prolonged hyperglycemia can cause non enzymatic glycosylation of proteins leading to conformational changes which have the capability of interference with their normal functions like activity of enzymes, capacity of degradation and recognition of the receptor. Moreover, interaction of these glycosylated proteins with macrophages which are derived from monocytes, endothelial cells and smooth muscle cells lead to inflammatory reactions and atherogenesis. These glycosylated proteins interact with their receptors and can increase oxidative stress, activation of protein kinase $\mathrm{C}$ with various changes in the expression of growth factors (8). Our study showed very weak positive correlation with FRS score and LDL cholesterol in T2DM patients with CAD. In one study, high FRS score had association with greater magnitude of cardiovascular disease of non-coronary nature and also simultaneously had association with greater magnitude of increased oxidized LDL cholesterols (9). Another study stated that levels of LDL cholesterol had poor association with cardiovascular risk stating that this lipoprotein being a poor predictor of cardiovascular risk in adults with advanced age (10) which is similar to our study finding. The odds ratio for increased oxidized LDL cholesterol with high risk of coronary heart disease risk before the onset of any coronary events was more than the individuals with already diagnosed coronary heart disease. This might be due to the fact that most of the patients might already be under statins supplements after the diagnosis of coronary heart disease. Our study showed weak positive correlation between ASCVD score and duration of T2DM. A study showed that patients having a history of more than 10 years of T2DM were more prone to develop CAD compared to those who had lesser duration of T2DM (11). Another study in China was conducted in two group of T2DM patients i.e mean age of 35 years with early onset of T2DM and mean age group of 55 years with late onset diabetes and were compared with non-fatal cardiovascular events. This stated that the possibility of developing cardiovascular events in future is 1.91 times more in the mean age group of 35 years patients having early onset of diabetes than mean age group of 55 years patients having late onset of diabetes (12). The weak positive correlation found in our study might be due to smaller sample size of T2DM patients suffering from CAD patients in comparison to T2DM patients without CAD. Our study showed weak negative correlation of BMI with ASCVD score in T2DM patients. A study showed that overweight or obese individuals had less cardiovascular risk in T2DM patients (13) which showed finding similar to our study. In a study T2DM patients who were grossly overweight and initiated insulin had $20 \%$ more risk of developing cardiovascular events after a duration of five years which was contrary to our study finding (14). The significant negative weak correlation between BMI and ASCVD score in our study might be due to the decrease in weight due to the consumption of oral hypoglycemic drugs for a prolonged period of time.

\section{Limitations}

This study being a cross-sectional study couldn't validate the reliability of the risk scores which could only be predicted in a cohort study.

\section{CONCLUSION}

Our observations imply that inclusion of important parameter such as duration of T2DM might improve the power of the CVD scores in T2DM patients. This may help us in better assessment of risk of CAD in T2DM patients. Future studies are needed to assess the performance of existing risk scores by including important parameter such as duration of diabetes 
which might help in improving these scoring systems in T2DM patients.

\section{ACKNOWLEDGEMENT}

We would like to thank all the associated personnel of the hospital who helped us during the study.

\section{CONFLICT OF INTEREST}

Conflict of interest was not found in this study.

\section{REFERENCES}

1. Einarson, T. R., Acs, A., Ludwig, C., Panton, U.H. Prevalence of cardiovascular disease in type 2 diabetes: a systematic literature review of scientific evidence from across the world in 2007-2017. Cardiovascular diabetology. 2018 Dec;17(1): 1-9.

2. Mohan, V., Venkatraman, J.V., Pradeepa, R. Epidemiology of cardiovascular disease in type 2 diabetes: the Indian scenario. Journal of diabetes science and technology. 2010 Jan;4(1): 158-170.

3. Aronson, D., Edelman, E. R. Coronary artery disease and diabetes mellitus. Cardiol Clin. 2014 Aug; 32(3): 439-455.

4. Garg, N., Muduli, S.K., Kapoor, A., Tewari, S., Kumar, S., Khanna, R., et al., Comparison of different cardiovascular risk score calculators for cardiovascular risk prediction and guideline recommended statin uses. Indian Heart Journal. 2017 Jul 1; 69(4): 458-463.

5. Fox, C. S., Sullivan, L., D’Agostino, R. B., Wilson, P. W. The significant effect of diabetes duration on coronary heart disease mortality: the Framingham Heart Study. Diabetes care. 2004 Mar 1; 27(3): 704-708.

6. Ye, X., Kong, W., Zafar, M. I., Chen, L. L. Serum triglycerides as a risk factor for cardiovascular diseases in type 2 diabetes mellitus: a systematic review and metaanalysis of prospective studies. Cardiovascular diabetology. 2019 Dec; 18(1): 1-10.

7. Levitzky, Y. S., Pencina, M. J., D’Agostino, R. B., Meigs, J. B., Murabito, J. M., Vasan, R. S., et al., Impact of impaired fasting glucose on cardiovascular disease: the Framingham Heart Study. Journal of the American College of Cardiology. 2008 Jan 22; 51(3): 264-270.

8. Singh, V. P., Bali, A., Singh, N., Jaggi, A. S. Advanced glycation end products and diabetic complications. Korean J Physiol Pharmacol. 2014 Feb; 18(1): 1-14.

9. Shepherd, J. Issues surrounding age: vascular disease in the elderly. Current opinion in lipidology. 2001 Dec 1; 12(6): 601-609.

10. Holvoet, P., Harris, T. B., Tracy, R. P., Verhamme, P., Newman, A. B., Rubin, S. M., et al., Association of high coronary heart disease risk status with circulating oxidized LDL in the well-functioning elderly: findings from the Health, Aging, and Body Composition study. Arteriosclerosis, thrombosis, and vascular biology. 2003 Aug 1;23(8): 1444-1448.

11. Rana, J. S., Liu, J. Y., Moffet, H. H., Jaffe, M., Karter, A. J. Diabetes and prior coronary heart disease are not necessarily risk equivalent for future coronary heart disease events. Journal of general internal medicine. 2016 Apr;31(4):387393.

12. Kianoush, S., Al Rifai, M., Whelton, S.P., Shaya, G.E., Bush, A.L., Graham, G., et al., Stratifying cardiovascular risk in diabetes: the role of diabetes-related clinical characteristics and imaging. Journal of Diabetes and its Complications. 2016 Sep 1;30(7):1408-1415.

13. Pagidipati, N.J., Zheng, Y., Green, J.B., McGuire, D.K., Mentz, R.J., Shah, S., et al., Association of obesity with cardiovascular outcomes in patients with type 2 diabetes and cardiovascular disease: Insights from TECOS. American heart journal. 2020 Jan 1; 219:47-57.

14. Anyanwagu, U., Mamza, J., Donnelly, R., Idris, I. Effects of obesity on metabolic and cardiovascular outcomes following insulin initiation in patients with type 2 diabetes. Obesity research \& clinical practice. 2018 Jan 1; 12(1): 72-84. 